\title{
O.P.E. and Power Corrections to the QCD coupling constant.
}

\author{
Ph. Boucaud ${ }^{\mathrm{a}}$, F. De Soto ${ }^{\mathrm{b}}$, A. Donini ${ }^{\mathrm{a}}$, J.P. Leroy ${ }^{\mathrm{a}}$, A. Le Yaouanc ${ }^{\mathrm{a}}$, J. Micheli ${ }^{\mathrm{a}}$, H. Moutarde ${ }^{\mathrm{a}}$, O. \\ Pène $^{\mathrm{a}}$, J. Rodríguez-Quintero ${ }^{\mathrm{c}}$
}

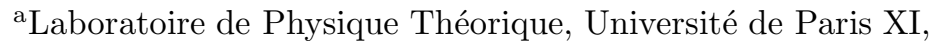
Batiment 210, 91405 Orsay-Cedex, France.

bDpto. de Física Atómica Molecular y Nuclear, Universidad de Sevilla. Avda. Reina Mercedes s/n, 41012 Sevilla, Spain.

cDpto. de F.A., E.P.S. La Rábida, Universidad de Huelva, 21819 Palos de la Fra., Spain.

Lattice data seems to show that power corrections should be convoked to describe appropriately the transition of the QCD coupling constant running from U.V. to I.R. domains. Those power corrections for the Landaugauge MOM coupling constant in a pure Yang-Mills theory $\left(N_{f}=0\right)$ are analysed in terms of Operator Product Expansion (O.P.E.) of two- and three-point Green functions, the gluon condensate $\left\langle A^{2}>\right.$ emerging from this study. The semi-classical picture given by instantons can be also used to look for into the nature of the power corrections and gluon condensate.

\section{The non-perturbative QCD coupling constant from lattice QCD.}

The non-perturbative calculation of the QCD coupling constant is a major open question, as it could give some insight into the problem of confinement. This calculation, as a very important task, has already been performed by several different methods, [1,2]. The method used here is based in the calculation of the coupling constant from the three gluon coupling [3], that allows an easier physical interpretation than the Schrödinger functional method, for example, and if it does not allow to study a quite wide range of energies, it will allow us to focus on the regime of energies where the transition from perturbative to non-perturbative regimes in QCD occurs.

The calculation of the coupling constant in this method comes from the lattice evaluation of two- and three-point gluon Green functions, the renormalised coupling being defined (according to MOM schemes) as the renormalised three gluon vertex where external propagators are explicitly amputated:

$g\left(k^{2}\right)=\frac{G^{(3)}\left(p_{1}^{2}, p_{2}^{2}, p_{3}^{2}\right)\left(Z_{3}\left(k^{2}\right)\right)^{3 / 2}}{G^{(2)}\left(p_{1}^{2}\right) G^{(2)}\left(p_{2}^{2}\right) G^{(2)}\left(p_{3}^{2}\right)}$,

where $G^{(2)}\left(p_{1}^{2}\right)$ and $G^{(3)}\left(p_{1}^{2}, p_{2}^{2}, p_{3}^{2}\right)$ are two- and three- point Green Function form factors [3, 1 , and $Z_{3}\left(k^{2}\right)=k^{2} G_{R}^{(2)}\left(k^{2}\right)$ is the gluon propagator renormalization constant.

\section{O.P.E. and $\left\langle A^{2}\right\rangle$ condensate.}

Lattice calculations of the QCD coupling constant suggest the necessity to add power corrections to the purely perturbative expressions, to correctly describe its running [4]. An Operator Product Expansion (O.P.E.) analysis of the Green functions in Landau gauge relates this power corrections to the existence of a non-perturbative $\left\langle A^{2}\right\rangle$ condensate [5], through expressions:

\footnotetext{
${ }^{1}$ In the lattice we will work in the minimum $A^{2}$ Landau gauge, $\partial_{\mu} A_{\mu}=0$ (Absolute Landau Gauge), so all gauge dependent quantities will be expressed in this particular gauge.
} 


$$
\begin{aligned}
G_{\text {O.P.E. }}^{(2)}\left(p^{2}\right) & =G_{\text {Pert. }}^{(2)}\left(p^{2}\right)+c \frac{\left\langle A^{2}\right\rangle_{R, \mu}}{p^{2}}, \\
\alpha_{s}^{\text {O.P.E }}\left(p^{2}\right) & =\alpha_{s}^{\text {Pert. }}\left(p^{2}\right)+c^{\prime} \frac{\left\langle A^{2}\right\rangle_{R, \mu}}{p^{2}},
\end{aligned}
$$

where perturbative expressions are developed at three loops, and the functions $c$ and $c^{\prime}$ include the Wilson coefficient of the expansion and the anomalous dimension of the condensate at leading logarithm.

By performing a combined fit of lattice results in a wide region of energies (from 3 to $10 \mathrm{GeV}$ ) to expressions in (2), in two different MOM schemes, a value of $\Lambda_{\overline{\mathrm{MS}}}$ is extracted, in fairly good agreement with the one obtained by the ALPHA collaboration [2], by a completely different method. A value of the $\left\langle A^{2}\right\rangle$ condensate comes out from the analysis.

The physical meaning of this condensate is still an open question, and a lot of work is being devoted to its study during last years, for example, in relation to confinement [6]. The aim in this work will be to study the possible semiclassical contribution to this condensate coming from instantons, and whether they might explain the presence of power corrections in Green Functions.

\section{The role of instantons.}

Instantons [7] are classical solutions of QCD equations of motion that have been longly studied as a possible description of QCD vacuum, and claimed to explain some non-perturbative properties of QCD at low energies, as the axial anomaly, $\eta^{\prime}$ mass, chiral condensate, etc (See [8] for a general overview).

Based in the fact that instantons are saddle points of QCD action, we could think of factorizing the path integral into an integral over semiclassical gauge field configurations (in this case instantons) and the integral of quantum fluctuations around this semi-classical background. This means that the gauge field $\left(A_{\mu}^{a}\right)$ could be decomposed into a instantonic background $\left(\left(A_{\mu}^{a}\right)_{\text {Inst. }}\right)$ plus quantum fluctuations:
$A_{\mu}^{a}=\left(A_{\mu}^{a}\right)_{\text {Inst. }}+Q_{\mu}^{a}\left(\left(A_{\mu}^{a}\right)_{\text {Inst. }}\right)$,

where in principle quantum fluctuations could deppend on the semi-classical background.

If we nevertheless assume the hypothesis that quantum fluctuations are not sensitive to the background [9], the O.P.E. $\left\langle A^{2}\right\rangle$-condensate, corresponding to the non-perturbative part of $\left\langle A^{2}\right\rangle$, will be:

$$
\left\langle A^{2}\right\rangle_{\text {O.P.E. }} \approx\left\langle\left(A_{\mu}^{a}\right)_{\text {Inst. }}^{2}\right\rangle \approx\left\langle A^{2}-Q^{2}(0)\right\rangle .
$$

The task in this work will be to compute the instantonic contribution to this condensate, that (up to other non-perturbative contributions, that could of course exist) we will finally compare to the O.P.E. one, computed in [5].

In order to compute $\left\langle A^{2}\right\rangle$ in an instantonic background, we will consider the gauge field in an ensemble of non-interacting $\mathrm{SU}(3)$ instantons (I) and anti-instantons $(\overline{\mathrm{I}})$ with radii $\left\{\rho_{i}\right\}$, centered in $\left\{z_{i}\right\}$, and with an orientation in color space given by $\left\{\left(O^{a \alpha}\right)_{i}\right\}$. If they are not interacting and randomly oriented, their contribution to the $\left\langle A^{2}\right\rangle$ condensate in the absolute Landau gauge would give:

$$
\left\langle A^{2}\right\rangle_{\mathrm{inst}} \approx \frac{N}{V} \int d^{4} x A_{\mu}^{a}(x) A_{\mu}^{a}(x)=12 \pi^{2} \rho^{2} n,
$$

where $A_{\mu}^{a}(x)$ is the standard 't Hooft Polyakov instanton gauge field [7], $\rho$ the average radius, and $n=\frac{N_{I}+N_{\bar{I}}}{V}$ the density.

If we accept the phenomenological values assigned to $n$ and $\rho$ by the Instanton Liquid Model [8] ( $n \sim 0.5 \mathrm{fm}^{-4}$ and $\left.\rho \sim 1 / 3 \mathrm{fm}\right)$, the instantonic contribution will be $\left\langle A^{2}\right\rangle_{\text {Inst. }} \sim 0.5 \mathrm{GeV}^{2}$. We will perform, however, our own analysis, thus testing the latter approach.

\subsection{Cooling.}

In principle, a direct measure of $A^{2}$ in the lattice should be possible, but the presence of the UV divergent part is hardly separable from the soft, instantonic one. The other possibility is to 
perform a cooling procedure, that, through a progressive elimination of UV fluctuations, will allow us to compute the number and size of instantons, giving an indirect measure of the $\left\langle A^{2}\right\rangle$ condensate through (5). Both methods agree after a high number of cooling sweeps, when quantum fluctuations are almost completely suppressed, but then cooling bias is so strong, as we will see, that no information can be recovered about the original situation.

We will use the traditional cooling method 10, even if it introduces a number of known biases, as I $-\bar{I}$ annihilation, and a modification of instanton sizes and lattice spacing. The approach proposed here is to compute instanton properties for different number of cooling sweeps, and extrapolate back to the thermalised situation [11], in order to recover their physical meaning $2^{2}$.

\subsection{Shape Recognition.}

Instantons will be localised in cooled lattices via a geometrical method (Described in [13].) that accepts a topological charge lump as an instanton when the ratio of the integral over lattice sites with topological charge bigger than a given fraction, $\alpha$, of the topological charge at the maximum and its theoretical counterpart,

$\epsilon(\alpha)=\frac{\int_{x / \frac{\left|Q_{\rho}(x)\right|}{\left|Q_{\rho}(0)\right|} \geq \alpha} d^{4} x Q_{\rho}(x)}{1-3 \alpha^{1 / 2}+2 \alpha^{3 / 4}}$,

is around 1 for a range of values of $\alpha$. To verify that it is really an instanton, we compute (6) both for the topologial charge density and the action, accepting it as an instanton only if it is self-dual.

Once the lump has been identified as an instanton, the radius will be computed from the size of the cluster where the integral has been developed, in agreement with the value of the radius deduced from the value of the topological charge at the maximum.

We have tested this method for different lattice spacings, from 0.055 to $0.23 \mathrm{fm}$. As a matter of fact, for big lattice spacings, instantons have radii

\footnotetext{
2 The use of improved cooling methods, as the one developed in $[12]$, could improve this approach, as radii evolution is minimised, but $\mathrm{I}-\overline{\mathrm{I}}$ annihilation is unavoidable, so the extrapolation will be anyway necessary.
}

of order of the lattice spacing, being in practice indistinguishable of UV fluctuations. Therefore, only lattices finer than $0.1 \mathrm{fm}$ will be used, otherwise an important number of instantons with small radii will be lost (all for radii below two lattice spacings).

\subsection{Results.}

If we compare the measures of the instanton density for two different lattice spacings, 0.074 and $0.101 \mathrm{fm}$, and different volumes, as a function of the number of cooling sweeps (Figure il), we can conclude that no finite volume effect affects our results, as the measures for the $24^{4}$ lattice and $32^{4}$ one are compatible. This is true excepting lattice volumes approaching the instanton volume.

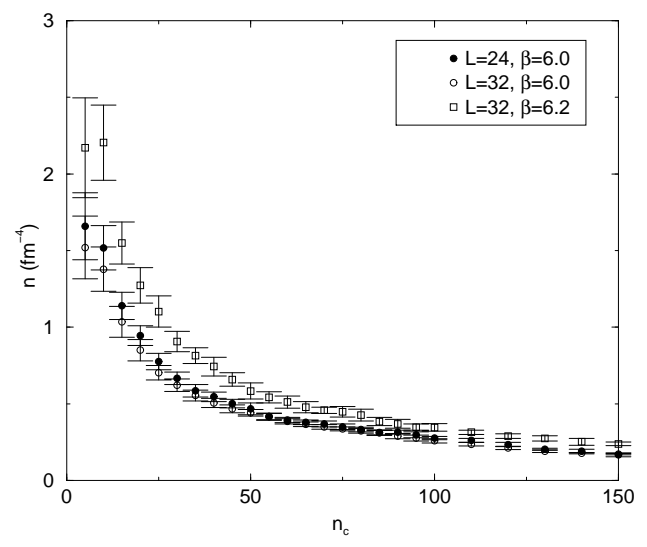

Figure 1. Density of instantons measured form different lattice volumes and lattice spacings $(a(\beta=$ $6.0)=0.101 \mathrm{fm}, a(\beta=6.2)=0.074 \mathrm{fm})$

For different values of the lattice spacing, on the contrary, bigger densities are found at the same number of cooling sweeps for finer lattices. In addition to the problems to detect instantons with small radii, there is a known problem of cooling procedures, they are not independent of the lattice spacing [10], and both effects could play an important role to explain the difference found between different lattice simulations. 
To illustrate this, if we fix the value of the density (that is, we perform a different number of cooling sweeps for each lattice spacing) and compare the average radii, they agree within the error limits. Moreover, comparing the distribution of instanton radii, they are in a quite good agreement (Figure 2), even with very few statistics (10 configuration each).

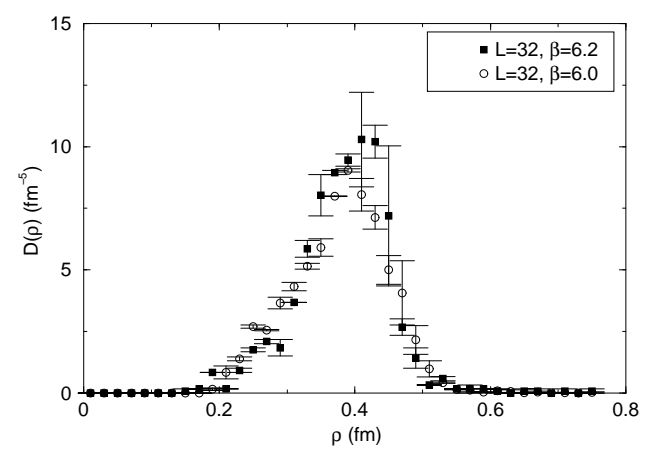

Figure 2. Comparison on instanton histogram at $n_{c}=10$ for $\beta=6.0$ and $n_{c}=15$ for $\beta=6.2$.

\subsection{A naive model of annihilation.}

With the method outlined above, we compute the density and size of instantons in a lattice, for different numbers of cooling sweeps, $n_{c}$, obtaining values with a strong dependence on $n_{c}$ (See figures), that avoids to obtain any physical information at fixed $n_{c}$. Moreover, this dependence on the number of cooling sweeps is different at different lattice spacing, so an extrapolation of results after cooling is unavoidable to obtain any physical information.

As a first approach to the understanding of this evolution, we will make a simple model, where instantons annihilate with antiinstantons (Being so $\Delta N=N_{I}-N_{\bar{I}}$ a constant) proportionally to their packing ratio, and to the number of antiinstantons, so that the equation for the evolution of $N=N_{I}+N_{\bar{I}}$ is:

$\frac{\partial N}{\partial n_{c}}=-\frac{\lambda}{2 V} \rho^{4}\left(n_{c}\right)\left(N\left(n_{c}\right)^{2}-\Delta N^{2}\right)$.
If we assumed $\rho\left(n_{c}\right)=$ cte, the solution of Eq. (7) would give $N\left(n_{c}\right) \sim \frac{N(0)}{1+\kappa n_{c}}$, the expression used in 13, as a first order approach. But our cooling procedure modifies instanton's size (See figure 3), in a way that we phenomenologically parametrize as:

$\rho\left(n_{c}\right)=\rho(0)\left(1+a \ln \left(1+n_{c}\right)\right)$.

We will include (8) in equation (7), with $\rho(0)$ the extrapolated radius at the thermalised situation and $a$ a constant to determine.

After performing a combined fit of our lattice results to the expressions (8) and the one coming from the integration of $(7)$, we can fix the initial values of the density, $n(0)$ and the radius $\rho(0)$, and the two constants that govern the evolution, $\lambda$ and $a$. An indirect measure of the $\left\langle A^{2}\right\rangle_{\text {Inst }}$ condensate is obtained.

Nevertheless, the result of the extrapolation is highly dependent on the value of $\rho(0)$, which due to its logarithmic behaviour is hardly reliable. We therefore prefer the value of $\left\langle A_{\text {Ins }}^{2}\right\rangle$ at the maximum of figure 3 as a crude estimation of the instantonic contribution to $\left\langle A^{2}\right\rangle$.

Having a slight dependence on the lattice spacing, the result given here for this quantity has to be understood as a first estimation, that would require for a further analysis [14].

\section{Conclussion.}

The result of the combined fit gives a value of the instantonic contribution to $\left\langle A_{\text {Ins }}^{2}\right\rangle \sim$ $0.4 \mathrm{GeV}^{2}$, however, as reasoned before, we will give the value at the maximum as a more reliable, although crude, estimation of the instantonic contribution to the $\left\langle A^{2}\right\rangle$ condensate. So our final result will be $\left\langle A_{\text {Ins }}^{2}\right\rangle=1.10(10) \mathrm{GeV}^{2}$ for the simulation at lattice spacing $0.101 \mathrm{fm}$, and $1.37(16) \mathrm{GeV}^{2}$ for the one at $0.074 \mathrm{fm}$.

This semiclassical evaluation of $\left\langle A^{2}\right\rangle$, which does not run with the scale, is difficult to relate to that appearing in the O.P.E. expansion, which does depend on the renormalisation scheme and scale. The typical scale of instantons is $\rho^{-1} \sim 0.7 \mathrm{GeV}$. Unluckly it is not possible to run 

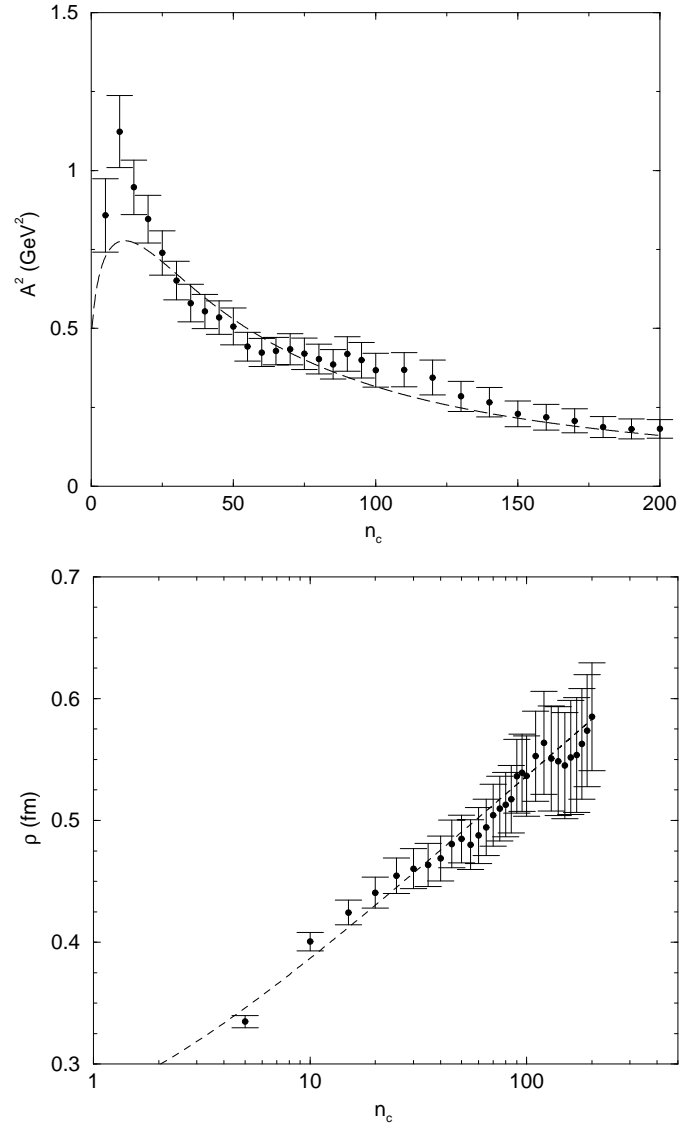

Figure 3. Results of the combined fit for the instanton density and radius as a function of the number of cooling sweeps for a $24^{4}$ lattice at $\beta=6.0$.

the $\left\langle A_{O \text { O.P.E. }}^{2}\right\rangle$ to such a low energy, where pertubative QCD is not valid. The lowest reacheble energy scale is $2.6 \mathrm{GeV}$ [5, 13], where the $\left\langle A^{2}\right\rangle$ condensate takes the value;

$$
\left\langle A_{\text {O.P.E. }}^{2}(2.6 \mathrm{GeV})\right\rangle=1.4(3)(3) \mathrm{GeV}^{2},
$$

the first error coming from the OPE determination of the condensate renormalised at $10 \mathrm{GeV}$, and the second from higher orders in the running.

Keeping in mind the level of uncertainty of these calculations, we can nevertheless claim a rather encouraging agreement between the in- stantonic contribution to the condensate and the one computed from the running of the Green Functions.

\section{Acknowledgements.}

F. de Soto wants to thank L.P.T. for its warm hospitality and Fundación Cámara and the MCYT (Contract BFM2002-03315) for financial support.

\section{REFERENCES}

1. C.T.H. Davies et al., Phys. Lett. B345 (1992) 42, Phys. Rev. D56 (1997) 2755, G.S. Bali and K. Schilling Phys. Rev. D47 (1993) 661.

2. S. Capitani et al., Nucl. Phys. Procc. Suppl. 63 (1998) 153; Nucl. Phys. B544 (1999) 669.

3. B. Alles et al., Nucl. Phys. B502 (1997) 325

4. Ph. Boucaud et al. J. High Energy Phys. 10 (1998) 017; D. Becirevic et al. Phys. Rev. D60 (1999) 094509.

5. Ph. Boucaud et al. Phys. Lett. B493 (2000) 315, F. De Soto and J. Rodríguez-Quintero Phys. Rev. D64 (2001) 114003.

6. K. I. Kondo, et al. prd652000085034, F.V. Gubarev, V.I. Zakharov, Phys. Lett. B501 (2001) 28.

7. G. 't Hooft, Phys. Rev. D14 (1976) 3432, [Erratum-ibid. Phys. Rev. D18 (1976) 2199].

8. T. Schafer and E. V. Shuryak, Rev. Mod. Phys. 70 (1998) 323.

9. $\mathrm{Ph}$. Boucaud et al., "A transparent expression of the $\mathrm{A}^{2}$-Condensate's renormalisation", to be published.

10. M. Teper, Phys. Lett. B 162, 357 (1985), Phys. Rev. D58 014505 (1998).

11. J. W. Negele, Nucl. Phys. Proc. Suppl. 73, 92 (1999) arXiv:hep-lat/9810053.

12. M. Garcia Perez, O. Philipsen and I. O. Stamatescu, Nucl. Phys. B 551, 293 (1999).

13. Ph. Boucaud et al., Phys. Rev. D 66 (2002) 034504.

14. Addendum to Phys. Rev. D 66 (2002) 034504, in preparation. 